\title{
Baseline tissue Doppler imaging-derived echocardiographic parameters and left ventricle reverse remodelling following cardiac resynchronization therapy introduction
}

Jerzy Wiliński, Danuta Czarnecka, Wiktoria Wojciechowska, Małgorzata Kloch-Badełek, Marek Jastrzębski, Bogumiła Bacior, Tomasz Sondej, Aleksander Kusiak

$1^{\text {st }}$ Department of Cardiology and Hypertension, Jagiellonian University Medical College, Krakow, Poland

Submitted: 13 December 2010

Accepted: 21 March 2011

Arch Med Sci 2011; 7, 5: 813-822

DOI: 10.5114/aoms.2011.25556

Copyright (c) 2011 Termedia \& Banach

\section{Abstract}

Introduction: The aim of the study was to assess the relation of baseline mechanical dyssynchrony with the left ventricular end-systolic volume (LVESV) decrease following cardiac resynchronization (CRT) therapy introduction.

Material and methods: Sixty consecutive patients (aged $66.3 \pm 8.7$ years; 57 men) with chronic heart failure ( $71.7 \%$ of ischaemic and $28.3 \%$ of non-ischaemic origin) and current indications for CRT were assessed before and 3 months after biventricular heart stimulator implantation. Longitudinal movements of twelve segments of the left ventricle (LV) (6 basal and 6 midlevel) and two segments of the right ventricle (RV) were analysed using tissue Doppler imaging (TDI) techniques with time from onset of $\mathrm{Q}$ wave in ECG to peak systolic velocity in colour-coded TDI ( $\left.T_{T D I}\right)$, time to peak strain $\left(T_{\text {strain }}\right)$ and time to peak strain rate $\left(T_{\text {strain rate }}\right)$. Minimal and maximal time differences within LV and between LV and RV walls were calculated.

Results: In the study group LVEF and 6-min walk test distance increased, while NYHA class, NT-proBNP level, left ventricular end-diastolic volume and LVESV decreased. Significant correlations between the magnitude of LVESV reduction with maximal time differences between $\mathrm{T}_{\text {strain }}$ of $12 \mathrm{LV}$ segments $(r=0.34$, $p=0.017)$ and time differences between $\mathrm{T}_{\mathrm{TDI}}$ basal LV-RV segments $(r=-0.29$, $p=0.041$ ) were found.

Conclusions: Only a few TDI-derived parameters such as maximal time differences between $T_{\text {strain }}$ of 12 LV segments and $T_{T D I}$ difference of LV-RV basal segments can be useful to predict the magnitude of left ventricle reverse remodelling after CRT introduction.

Key words: heart failure, pacemakers, echocardiography, Doppler dyssynchrony.

\section{Introduction}

Cardiac resynchronization therapy (CRT) has a well-established position in the treatment of drug-refractory chronic heart failure (CHF) [1, 2]. The CRT improves filling of the left ventricle, increases ejection fraction (LVEF), cardiac output and systolic blood pressure, reduces mitral insufficiency and, in long-term observations, induces left ventricle reverse remodelling. The CRT was also found to decrease sleep-related breathing disorders that

\author{
Corresponding author: \\ Jerzy Wiliński MD, PhD \\ $1^{\text {st }}$ Department of Cardiology \\ and Hypertension \\ Jagiellonian University \\ Medical College \\ 17 Kopernika \\ 31-501 Krakow, Poland \\ Phone: +48 0124247300 \\ Fax: +480124247320 \\ E-mail: putamen@interia.pl
}


augment $\mathrm{CHF}$, improve the function of the autonomic nervous system and lead to a fall of some inflammatory markers [3-6]. Solid evidence from clinical trials has proven that CRT amends physical capacity and quality of life, and decreases morbidity and mortality related to CHF. Unfortunately, a considerable proportion (30-40\%) of individuals undergoing CRT in clinical trials did not benefit from this novel therapy [7]. Since the absence of echocardiographic dyssynchrony is associated with a less favourable CRT outcome, the answer to the question of CRT failure is also believed to be found in the complex heart mechanical performance [8]. A debate on the issue has been triggered with echocardiographic researchers from around the world contributing volubly to the discussion.

The aim of this study was to assess the relation of baseline mechanical dyssynchrony in the echocardiographic study with the use of tissue Doppler imaging (TDI), strain and strain rate parameters with the left ventricle reverse remodelling observed after CRT implantation, a powerful long-term prognostic factor, in typical daily practice $\mathrm{CHF}$ patients receiving biventricular heart stimulation $[9,10]$. A unique approach in the analysis of CRT outcome was engaged: the presence of at least $10 \%$ relative left ventricular end-systolic volume (LVESV) reduction or the magnitude of LVESV decrease following biventricular heart stimulator was considered.

\section{Material and methods}

\section{Study group}

Seventy-two consecutive patients with chronic heart failure in NYHA class III-IV stable for at least 3 months despite optimized pharmacotherapy, with left ventricle end-diastolic diameter (LVEDd) $>55 \mathrm{~mm}$, left ventricular ejection fraction (LVEF) $\leq 35 \%$ and wide QRS complex ( $\geq 120 \mathrm{~ms}$ ), were involved in the prospective study. Eight individuals met the exclusion criteria, two refused to have CRT introduced, and in another two implantation of the left ventricular lead did not succeed. Eventually 60 patients (aged $66.3 \pm 8.7$ years, 57 men [95\%], 3 women [5\%]) participated in the study.

\section{Exclusion criteria}

Exclusion criteria comprised the presence of unstable angina, acute myocardial infarction, coronary artery bypass graft or percutaneous coronary intervention within 3 months; continuous or intermittent intravenous inotropic drug therapy, an estimated life expectancy of less than 12 months, a mechanical right-side heart valve, heart transplant, pregnancy or concurrent enrolment in a study thought to confound the results.

\section{Study design}

Before CRT implantation and at 3 months of the follow-up (12-16 weeks) individuals were evaluated clinically with NYHA class, 6-min walk test (6-MWT), history with questions about hospitalizations and echocardiographically. Criteria of diagnosis of different clinical and biochemical disorders were adopted from the European Society of Cardiology (ESC) guidelines [1]. All patients undergoing CRT had had coronarography done. Ischaemic background of CHF was diagnosed when there was at least $50 \%$ stenosis of one or more coronary artery branches or a patient had a history of coronary artery bypass graft (CABG) or percutaneous coronary intervention $(\mathrm{PCI})[11,12]$.

Informed consent was obtained from each patient. The study protocol conforms to the ethical guidelines of the 1975 Declaration of Helsinki and was accepted by the Jagiellonian University Medical College Bioethical Committee.

\section{Biventricular device implantation}

Patients in sinus rhythm received an atriobiventricular stimulator (DDDR BiV $-n=47$ ), while individuals with permanent atrial fibrillation received a biventricular device (VVIR BiV $-n=13)$. Patients with a history of cardiac arrest and/or malignant ventricular arrhythmias had the system with combined cardioverter-defibrillator (CRT-D) implanted (18 cases). During CRT all leads were implanted transvenously. The left ventricular lead, guided by a venogram, was placed in a coronary sinus tributary in a stable lateral or postero-lateral position, with $a<3.5 \mathrm{~V}$ capture threshold. The right ventricular lead was placed in the septal or outflow tract (RVOT) position. Positions of lead tips were verified on frontal and sagittal chest $X$-ray. Two patients required left ventricular lead repositioning due to their dislocation. Atrioventricular (AV) delay remained standard programmed 150/120 ms, unless conduction of cardiotopic systoles was preserved. In those patients $\mathrm{AV}$ was shortened until ventricles were paced (5 cases). Interventricular (VV) timing left nominal $5 \mathrm{~ms}$, unless no signs of biventricular stimulation in the body surface ECG were observed. $\mathrm{VV}$ was changed then to elicit the picture of QRS fusion beats in ECG lead V1 (7 cases) like in the study of Bailey et al. [13]. Such a VV-delay optimization was there proven to correlate better with maximum rate of rise of left ventricular pressure $\left(\mathrm{d} p / \mathrm{d} t_{\max }\right)$ than the strategy to obtain the narrowest paced QRS complexes.

In patients with permanent atrial fibrillation, ventricles' rate control with $\beta$-blockers and digoxin was assessed. In one patient an unsatisfactory pharmacologic effect ( $<95 \%$ of biventricular stimulation) was observed and ablation of the 
atrioventricular junction was performed. The followup was prolonged over 3 months after the procedure.

\section{Echocardiography}

Echocardiographic examinations were performed with the apparatus Vivid 7 (General Electric Healthcare). Left ventricular volumes and LVEF were evaluated using biplane Simpson's method. All stored echo recordings were analysed off-line by means of the Echo Pack system with GE brand software by an experienced physician with respect to the echocardiography norms of the European Association of Echocardiography and recommendations of the American Society of Echocardiography (ASE) for performance and reporting of echocardiographic studies for CRT $[14,15]$. A different approach from the ASE recommendations regarded four aspects. Patients with atrial fibrillation were included - analysis of atrial fibrillation was done on at least 5-beat sequences of 60-80 bpm rate. The search for the desired peak TDI, strain rate and strain was not limited to the parts of curves within the time when the aortic valve was open. Multipeak curves with peaks of similar height were excluded from the analysis. Each single myocardial wall was recorded separately (not in sets of two opposite walls) in order to record longitudinal segments' movements along scan lines and reduce the Doppler angle dependent bias.

Longitudinal movements of twelve segments of the left ventricle (LV) (6 basal and 6 midlevel) and two segments of the right ventricle (RV) were analysed using tissue Doppler imaging (TDI) techniques with times from onset of $\mathrm{Q}$ wave in $\mathrm{ECG}$ to peak systolic velocity in colour-coded TDI ( $\left.T_{T D I}\right)$, time to peak strain $\left(T_{\text {strain }}\right)$ and time to peak strain rate $\left(T_{\text {strain rate }}\right)$. Standard deviations (SD) of times of LV segments were calculated, and minimum and maximum time differences among LV segments as well as LV and RV walls in these different techniques were analysed.

The following dyssynchrony indices were calculated:

1) T-SD - standard deviation of time from the beginning of QRS complex to maximum systolic velocity/strain/strain rate among 12 left ventricle segments,

2) T-12 - maximum difference of time from the beginning of QRS complex to maximum systolic velocity/strain/strain rate among 12 left ventricle segments (basal and midlevel),

3) T-6-basal - maximum difference of time from the beginning of QRS complex to maximum systolic velocity/strain/strain rate among 6 basal left ventricle segments,
4) T-IVS- free wall - maximum difference of time from the beginning of QRS complex to maximum systolic velocity/strain/strain rate between basal and midlevel septal segments (septal and anteroseptal segments) and left ventricle free wall (lateral and posterior segments),

5) T-basal IVS-free wall - maximum difference of time from the beginning of QRS complex to maximum systolic velocity/strain/strain rate between basal septal segments (septal and antero-septal segments) and left ventricle free wall (lateral and posterior segments),

6) T-IVD - maximum difference of time from the beginning of QRS complex to maximum systolic velocity/strain/strain rate between basal left ventricle and right ventricle segments.

\section{Echocardiographic recordings interpretation limitations}

Poor image quality and the ambiguity of interpretation of the obtained curves caused that $26.4 \%$ of TDI, strain rate and strain parameters were incalculable. Based on the analysis of 10 randomly chosen recordings, intra-observer variability for LVESV estimation was $4 \pm 3 \%$, for peak systolic velocity $-7 \pm 10 \%$, for peak systolic strain $-9 \pm 12 \%$ and for peak systolic strain rate $-9 \pm 11 \%$.

\section{The CRT outcome assessment}

Patients with at least $10 \%$ relative reduction of LVESV after 3 months of the follow-up were classified as responders to CRT. Clinical and echocardiographic parameters were compared between these two groups. In the second part of the analysis correlations between TDI-derived variables and the magnitude of reverse remodelling as a CRT outcome measure were appraised.

\section{Statistical analysis}

Statistical analysis was performed within SAS System 9.1 (SAS Institute Inc., Cary North Carolina, USA) by Student's $t$-test, Fisher's exact test and $\chi^{2}$ test. The PROC CORR procedure was applied to assess correlations. Statistical significance was considered when $p<0.05$. Data are expressed as mean $( \pm$ SD) for continuous variables and as absolute frequencies or relative percentages for categorical variables.

\section{Results}

\section{Clinical characteristics of the study group}

In the study group $61.6 \%$ were in sinus rhythm, $16.7 \%$ had paroxysmal atrial fibrillation and $21.7 \%$ permanent fibrillation. Conduction disorders constituted left bundle branch block (65.0\%), right bundle branch block (1.7\%), and ventricular 
conduction disturbances of other morphology (33.3\%). Two patients with previously implanted ventricular heart stimulator (VVI), 9 with dual chamber pacemaker (DDD) and 3 with dual chamber implantable cardioverter-defibrillator (DDD-ICD) had their systems upgraded to CRT. Clinical characteristics of the study group are summarized in Table I.

\section{Course of the study}

Three patients died in the 3-month-follow-up: one of stroke, one of myocardial infarction, and one suddenly at home of unknown cause. These individuals were excluded from the further analysis. In the observation period 10 patients were hospitalized, 7 of them due to CHF exacerbation. All prescriptions (medications - see Table I) remained stable through the study.

\section{Effects of CRT}

In the whole study group LVEF increased, 6-MWT distance rose, and left ventricular end-diastolic volume (LVEDV) and LVESV decreased. Mean NYHA class and QRS width diminished (Table II). Results of specialized examinations before and after implementation in the studied subgroups of responders ( $n=34,59.7 \%)$ and non-responders to CRT $(n=23,40.3 \%)$ are shown in Table III.

\section{Clinical and classic echocardiographic features}

Responders did not differ from non-responders in respect of baseline left ventricular end-diastolic and end-systolic diameters and volumes, or the size of mitral regurgitation (Table III).

\section{Baseline dyssynchrony echocardiographic indices and CRT outcome}

In the analysis of colour-coded TDI, myocardial strain rate and strain indices, the only parameter that differentiated the groups with and without reverse remodelling was $T_{\text {strain }}$ of $12 \mathrm{LV}$ segments (Table IV). Significant correlations between the magnitude of LVESV reduction with maximal time differences between $T_{\text {strain }}$ of $12 \mathrm{LV}$ segments and time differences between $T_{T D I}$ basal LV-RV segments were found (Table V, Figure 1).

\section{Multivariate analysis}

In the multivariate analysis, variables that differentiated the groups with and without $\geq 10 \%$

Table I. Baseline clinical data of the whole study group and of responders and non-responders subgroups according to the applied criterion of at least $10 \%$ end-systolic left ventricular volume reduction at 3-months of follow-up (data presented as mean value with standard deviation (SD) or number of patients with the percentage share (\%) of the whole analysed group or subgroup, $p$-value for comparison between responders and non-responders)

\begin{tabular}{|c|c|c|c|c|}
\hline Parameter & All $(n=60)$ & Responders $(n=34)$ & Non-responders $(n=23)$ & Value of $p$ \\
\hline Age [years] & $66.3(8.7)$ & $65.8(8.2)$ & $67.1(9.4)$ & 0.60 \\
\hline Gender - male [\%] & $57(95 \%)$ & $33(97 \%)$ & $24(92 \%)$ & 0.57 \\
\hline $\mathrm{BMI}\left[\mathrm{kg} / \mathrm{m}^{2}\right]$ & $26.1(4.3)$ & $26.0(3.0)$ & $27.0(5.0)$ & 0.33 \\
\hline NYHA & $3.1(0.2)$ & $3.1(0.3)$ & $3.1(0.2)$ & 0.91 \\
\hline Ischaemic aetiology of CHF & $43(71 \%)$ & $21(62 \%)$ & $22(84 \%)$ & 0.04 \\
\hline Permanent atrial fibrillation & $13(22 \%)$ & $6(18 \%)$ & $7(27 \%)$ & 0.048 \\
\hline Diabetes type 2 & $25(42 \%)$ & $12(35 \%)$ & $13(50 \%)$ & 0.30 \\
\hline Chronic obstructive pulmonary disease & $11(18 \%)$ & $7(21 \%)$ & $4(15 \%)$ & 0.52 \\
\hline Hypercholesterolaemia & $46(76 \%)$ & $26(76 \%)$ & $20(76 \%)$ & 0.99 \\
\hline Anaemia & $2(3 \%)$ & $2(6 \%)$ & $0(0 \%)$ & 0.35 \\
\hline CRT-D & $18(30 \%)$ & $11(32 \%)$ & $7(27 \%)$ & 0.56 \\
\hline History of stroke & $4(6.7 \%)$ & $3(9 \%)$ & $1(3 \%)$ & 0.62 \\
\hline Chronic renal disease & $20(33.3 \%)$ & $8(23 \%)$ & $12(46 \%)$ & 0.09 \\
\hline \multicolumn{5}{|l|}{ Medication } \\
\hline ACEI/ARB & $51(85 \%)$ & $30(88 \%)$ & $21(81 \%)$ & 0.48 \\
\hline$\beta$-Blocker & $58(96.7 \%)$ & $33(97 \%)$ & $25(96 \%)$ & 0.99 \\
\hline Loop diuretic & $53(88.3 \%)$ & $28(82 \%)$ & $25(96 \%)$ & 0.12 \\
\hline Potassium-sparing diuretic & $40(66.7 \%)$ & $25(73 \%)$ & $15(58 \%)$ & 0.27 \\
\hline Digoxin & $13(21.7 \%)$ & $8(23 \%)$ & $5(19 \%)$ & 0.76 \\
\hline Amiodarone & $18(30 \%)$ & $9(26 \%)$ & $9(35 \%)$ & 0.39 \\
\hline
\end{tabular}

CRT-D - cardiac resynchronization therapy defibrillator, ACEI - angiotensin converting enzyme inhibitor, ARB - angiotensin receptor blocker 
Table II. Clinical and echocardiographic parameters in the study group before and 3 months after CRT introduction

\begin{tabular}{|lccc|}
\hline Parameter & Before CRT $(n=57)$ & 3 months after CRT $(n=57)$ & Value of $p$ \\
\hline NYHA & $3.1(0.2)$ & $2.2(0.7)$ & $<0.001$ \\
\hline 6-MWT [m] & $298.0(107.4)$ & $373.1(127.1)$ & $<0.001$ \\
\hline LVEDd [mm] & $73.3(8.9)$ & $71.5(9.8)$ & 0.003 \\
\hline LVESd [mm] & $62.4(10.0)$ & $60.6(11.3)$ & 0.034 \\
\hline LVEDV [ml] & $244.3(83.8)$ & $226.4(88.6)$ & 0.002 \\
\hline LVESV [ml] & $192.8(71.9)$ & $168.7(76.5)$ & $<0.001$ \\
\hline SV [ml] & $51.5(16.6)$ & $57.7(16.9)$ & $<0.001$ \\
\hline LVEF [\%] & $21.7(4.8)$ & $26.0(4.8)$ & $<0.001$ \\
\hline NT-proBNP [pg/ml] & $2387.2(1836.1)$ & $1844.5(1312.3)$ & 0.027 \\
\hline QRS width & $184.2(28.3)$ & $152.7(19.1)$ & $<0.001$ \\
\hline
\end{tabular}

NYHA - New York Heart Association functional class, 6-MWT - 6-min walk test distance, LVEDd - left ventricular end-diastolic diameter, LVESd - left ventricular end-systolic diameter, LVEDV - left ventricular end-diastolic volume, LVESV - left ventricular end-systolic volume, SV - stroke volume, LVEF-left ventricular ejection fraction, NT-proBNP-N-terminal pro-B-type natriuretic peptide

Table III. Specialized examinations results of responders and non-responders according to the applied criterion of at least 10\% end-systolic left ventricular volume reduction at 3-month follow-up

\begin{tabular}{|lcccccc|}
\hline Parameter & \multicolumn{3}{c}{ Responders, $n=34$ (59.6\%) } & \multicolumn{2}{c|}{ Non-responders, $n=23(40.4 \%)$} \\
\cline { 2 - 7 } & Initially & After 3 months & Value of $p$ & Initially & After 3 months & Value of $p$ \\
\hline LVEDd [mm] & $73.2(7.4)$ & $69.9(8.3)$ & $<0.001$ & $73.5(10.9)$ & $73.9(11.5)$ & 0.47 \\
\hline LVESd [mm] & $62.0(7.9)$ & $58.1(9.4)$ & 0.003 & $63.0(12.7)$ & $64.2(13.0)^{\star}$ & 0.13 \\
\hline LVEDV [ml] & $246.3(61.1)$ & $209.5(58.7)$ & $<0.001$ & $241.3(110.7)$ & $251.4(117.6)$ & 0.054 \\
\hline LVESV [ml] & $194.5(55.9)$ & $153.0(51.4)$ & $<0.001$ & $190.3(92)$ & $191.8(99.9)$ & 0.68 \\
\hline SV [ml] & $51.6(12.8)$ & $56.3(12.4)$ & 0.002 & $51.4(21.3)$ & $59.6(22.2)$ & 0.002 \\
\hline MR [\%] & $25.6(14)$ & $18.4(11)$ & 0.001 & $23.7(11)$ & $24.4(11)$ & 0.62 \\
\hline QRS [ms] & $179(28)$ & $153(20)$ & 0.001 & $199(27)$ & $152(17)$ & 0.001 \\
\hline LVEF [\%] & $21.5(4.9)$ & $27.1(4.8)$ & $<0.001$ & $21.9(4.7)$ & $24.5(4.7)^{\star}$ & 0.003 \\
\hline 6-MWT [m] & $315.7(112.1)$ & $392.3(109.5)$ & $<0.001$ & $271.9(96)$ & $344.7(147.4)$ & 0.001 \\
\hline NYHA & $3.1(0.3)$ & $2.1(0.5)$ & $<0.001$ & $3.1(0.2)$ & $2.5(0.8)$ & 0.001 \\
\hline NT-proBNP [pg/ml] & 2005.6 & 1512.0 & 0.012 & 3150.5 & 2509.5 & 0.32 \\
& $(1632.3)$ & $(1148.7)$ & & $(2034.3) \#$ & $(1403.6)^{*}$ & \\
\hline VV-sep [cm] & & $8.1(2.9)$ & & & $8.9(2.3)$ & \\
\hline
\end{tabular}

6-MWT - 6-min walk test distance, LVEDd - left ventricular end-diastolic diameter, LVEDV - left ventricular end-diastolic volume, LVEF - left ventricular ejection fraction, LVESd - left ventricular end-systolic diameter, LVESV - left ventricular end-systolic volume, MR - mitral regurgitation - presented as \% of left atrium area, NT-proBNP - N-terminal pro-B-type natriuretic peptide, NYHA - New York Heart Association Functional Classification, VV-sep. - right and left ventricular electrode tips' separation on sagittal chest $X$-ray

Each parameter is presented as arithmetic mean with standard deviation (SD), $p$ - for the difference between initial and after 3-month followup values of the same parameter, $\# p<0.05$ - for the difference between initial parameters of responders and non-responders, ${ }^{*} p<0.05-$ for the difference between parameters of responders and non-responders measured after 3 months of follow-up

LVESV reduction in the follow-up were included: an ischaemic aetiology of $\mathrm{CHF}$, permanent atrial fibrillation, prevalence of hypertension, hypotension and $\mathrm{N}$-terminal pro-B-type natriuretic peptide (NT-proBNP) concentration [16]. Also left bundle branch block (LBBB) and non-LBBB morphology of QRS complex were considered. The predictive values of $T_{\text {strain }}$ of $12 \mathrm{LV}$ segments and $T_{T D I}$ basal LV-RV regarding LVESV reduction lost their statistical significance.

\section{Discussion}

Significance of response to CRT criteria choice. Rationale for the magnitude of LVESV reduction assessment as CRT outcome measure

Response to CRT criteria hugely influence study results. Moreover, response to CRT rates differ dramatically depending on the applied definition. Compiling data from the literature suggest that reversal of the maladaptive left ventricle remodelling 
Table IV. Baseline parameters [ms] of mechanical dyssynchrony assessed with colour-coded tissue Doppler imaging (TDI), myocardial strain and strain rate in the whole study group, responders and non-responders according to the applied criterion of at least $10 \%$ end-systolic left ventricular volume reduction at 3-month follow-up

\begin{tabular}{|c|c|c|c|c|}
\hline Parameter & All & Responders & Non-responders & Value of $p$ \\
\hline \multicolumn{5}{|l|}{ Colour-coded TDI } \\
\hline $\mathrm{T}_{\mathrm{TDI}}-\mathrm{SD}$ & $58.0(16)$ & $54.8(18)$ & $62(12.8)$ & 0.14 \\
\hline $\mathrm{T}_{\mathrm{TDI}}-12$ & $176.2(54)$ & $165.8(62)$ & $189.1(41)$ & 0.15 \\
\hline $\mathrm{T}_{\mathrm{TDI}}$-6-basal & $153.2(96)$ & 159.5 (111) & $143.8(66)$ & 0.55 \\
\hline $\mathrm{T}_{\mathrm{TDI}}$-IVS-free wall & $222.8(181)$ & $200(180)$ & $258.3(183)$ & 0.29 \\
\hline $\mathrm{T}_{\mathrm{TDI}}$-basal IVS-free wall & $130.9(88)$ & $126.8(85)$ & $137.4(96)$ & 0.69 \\
\hline $\mathrm{T}_{\left.\mathrm{TDI}\right|^{-I V D}}$ & $59.4(46)$ & $49.5(40)$ & $73.7(51)$ & 0.06 \\
\hline \multicolumn{5}{|l|}{ Strain } \\
\hline$T_{\text {strain }}-S D$ & $125.2(33)$ & $131.83(30)$ & $115.6(36)$ & 0.09 \\
\hline $\mathrm{T}_{\text {strain }}-12$ & 414.6 (111) & 441.9 (114) & 367.7 (89) & 0.023 \\
\hline $\mathrm{T}_{\text {strain }}-6$-basal & 318.9 (109) & $342.2(108)$ & $287.5(106)$ & 0.09 \\
\hline $\mathrm{T}_{\text {strain }}$-IVS-free wall & $391.2(243)$ & $405.9(228)$ & $495.3(269)$ & 0.60 \\
\hline $\mathrm{T}_{\text {strain-basal IVS-free wall }}$ & 210.7 (139) & $229.0(141)$ & $180(135)$ & 0.22 \\
\hline$T_{\text {strain }}-I V D$ & $169.3(103)$ & $161.5(100)$ & $179.5(108)$ & 0.56 \\
\hline \multicolumn{5}{|l|}{ Strain rate } \\
\hline $\mathrm{T}_{\text {strain rate }} \mathrm{SD}$ & $133.4(29)$ & $140.6(27)$ & $124.7(31)$ & 0.06 \\
\hline$T_{\text {strain rate }}-12$ & $403.8(98)$ & $418.3(100)$ & $384.1(94)$ & 0.22 \\
\hline $\mathrm{T}_{\text {strain rate }}-6$-basal & $341.9(78)$ & $359.6(68)$ & $318(87)$ & 0.07 \\
\hline $\mathrm{T}_{\text {strain rate }}-$ IVS-free wall & $454.7(283)$ & $494.1(306)$ & $395.5(242)$ & 0.25 \\
\hline $\mathrm{T}_{\text {strain rate }}$-basal IVS-free wall & $243.6(144)$ & $271.7(141)$ & $192.5(140)$ & 0.08 \\
\hline $\mathrm{T}_{\text {strain rate }} \mathrm{IVD}^{-\mathrm{V}}$ & $169.2(116)$ & $177.5(124)$ & $158.6(108)$ & 0.58 \\
\hline
\end{tabular}

$T$-SD - standard deviation of time from the beginning of QRS complex to maximum systolic velocity/strain/strain rate in 12 left ventricle segments, T-12 - maximum difference of time from the beginning of QRS complex to maximum systolic velocity/strain/strain rate among 12 left ventricle segments (basal and midlevel), T-6-basal - maximum difference of time from the beginning of QRS complex to maximum systolic velocity/strain/strain rate among 6 basal left ventricle segments, T-IVS- free wall-maximum difference of time from the beginning of QRS complex to maximum systolic velocity/strain/strain rate between basal and midlevel septal segments (septal and antero-septal segments) and left ventricle free wall (lateral and posterior segments), T-basal IVS-free wall - maximum difference of time from the beginning of QRS complex to maximum systolic velocity/strain/strain rate between basal septal segments (septal and antero-septal segments) and left ventricle free wall (lateral and posterior segments), T-IVD - maximum difference of time from the beginning of QRS complex to maximum systolic velocity/strain/strain rate between basal left ventricle and right ventricle segments

mechanism in CHF observed a few months after CRT implementation is the best predictor of positive long-term outcome. Yu et al. showed in a series of 141 CRT patients that reduction of LVESV of at least $10 \%$ was related to a very low event rate $(6.9 \%$ allcause mortality) in a mean follow-up period of 695 days [9]. Di Biase et al. in an analysis from a prospective registry including 398 consecutive patients, with the median duration of follow-up of 4.4 years, demonstrated that the magnitude of reverse remodelling measured by LVEF and LVESV after 3 months of CRT implementation irrespectively of aetiology predicted outcome [10].

\section{Methodological considerations}

Our analyses of the colour-coded TDI velocities, strain and strain rate curves were not restrained to the ejection period. This common practice distorts dyssynchrony imaging since contraction of certain left ventricle parts takes place not only during the ejection phase but also before opening and after the aortic valve closure. Such velocities may be greater than the ejection peaks and need to be distinguished from passive tethering by the adjoining myocardial segments. Both these movements are interchangeably classified by different authors as the phenomenon of postsystolic shortening/thickening (PSS). The PSS is therefore commonly observed in patients undergoing CRT, was found to be of high sensitivity in detecting ischaemia in other studies, but might be present even in one third of left ventricle segments in healthy individuals [18]. A problem also arises when fine tuning of the myocardial region of interest during TDI analysis fails to produce a single 
reproducible peak. Strategies of proceeding in such situations varied in different studies, and the ASE recommendation of choosing the earlier peak if two or more peaks are of the same height is also challenged. In our research multi-peak curves with comparable maximal height were excluded from the analysis. As described in the 'material and methods' section, using the quality experience of previous studies, the offline calculations were performed by one experienced physician with the variability of the studied TDI, strain and strain rate parameters not exceeding $10 \%$, which has to be considered as an attribute of this paper [19, 20].

One of our study assumptions was to provide information useful for typical CHF patients undergoing CRT. It is crucial to remember that, although presence of permanent atrial fibrillation is believed to predict poorer outcome, the huge meta-analysis of Upadhyay et al. of 1,164 CRT individuals indicates that the strategy, undertaken also in our study, of ventricles' rate control with $\beta$-blockers, digoxin and amiodarone and, if ineffective, ablation of the atrioventricular node confers a benefit similar to that of CRT to the group of patients in sinus rhythm [2, 21]. Despite reservations about the accuracy of echocardiographic analysis in atrial fibrillation patients, some researchers appreciate the significance of mechanical dyssynchrony in this group and successfully apply its quantification in their studies $[22,23]$.

\section{Efficiency of colour-coded TDI derived indices of baseline dyssynchrony and interventricular dyssynchrony appraisal}

The largest body of literature and the principal method in clinical use to quantify mechanical dyssynchrony in CHF patients undergoing CRT is
Table V. Correlation between the magnitude of LVESV reduction $[\mathrm{ml}$ ] following $C R T$ introduction and initial parameters of mechanical dyssynchrony [ms] assessed with colour-coded tissue Doppler imaging (TDI), myocardial strain and strain rate in the whole study group

\begin{tabular}{|c|c|c|}
\hline Parameter & Value of $r$ & Value of $p$ \\
\hline \multicolumn{3}{|l|}{ Colour-coded TDI } \\
\hline $\mathrm{T}_{\mathrm{TDI}}-\mathrm{SD}$ & -0.05 & 0.72 \\
\hline $\mathrm{T}_{\mathrm{TDI}^{-12}}$ & -0.07 & 0.63 \\
\hline $\mathrm{T}_{\mathrm{TDI}^{-6}}{ }^{-6}$-basal & -0.03 & 0.80 \\
\hline $\mathrm{T}_{\mathrm{TDI} I^{-I V S} \text {-free wall }}$ & -0.16 & 0.28 \\
\hline $\mathrm{T}_{\mathrm{TDI}}$-basal IVS-free wall & -0.04 & 0.76 \\
\hline $\mathrm{T}_{\mathrm{TDI}}{ }^{-\mathrm{IVD}}$ & -0.29 & 0.041 \\
\hline \multicolumn{3}{|l|}{ Strain } \\
\hline$T_{\text {strain }}-S D$ & 0.18 & 0.24 \\
\hline $\mathrm{T}_{\text {strain }}-12$ & 0.34 & 0.017 \\
\hline $\mathrm{T}_{\text {strain }}-6$-basal & 0.21 & 0.16 \\
\hline $\mathrm{T}_{\text {strain }}$-IVS-free wall & 0.15 & 0.30 \\
\hline $\mathrm{T}_{\text {strain }}$-basal IVS-free wall & 0.07 & 0.64 \\
\hline $\mathrm{T}_{\text {strain }}-\mathrm{IVD}$ & 0.05 & 0.76 \\
\hline \multicolumn{3}{|l|}{ Strain rate } \\
\hline $\mathrm{T}_{\text {strain rate }}-\mathrm{SD}$ & 0.19 & 0.19 \\
\hline $\mathrm{T}_{\text {strain rate }}-12$ & 0.20 & 0.16 \\
\hline $\mathrm{T}_{\text {strain rate }}-6$-basal & 0.14 & 0.34 \\
\hline $\mathrm{T}_{\text {strain rate }}{ }^{-I V S}$-free wall & 0.15 & 0.33 \\
\hline $\mathrm{T}_{\text {strain rate-basal IVS-free wall }}$ & 0.08 & 0.60 \\
\hline $\mathrm{T}_{\text {strain rate }}-\mathrm{IVD}$ & 0.03 & 0.81 \\
\hline
\end{tabular}

Abbreviations - see Table IV
A

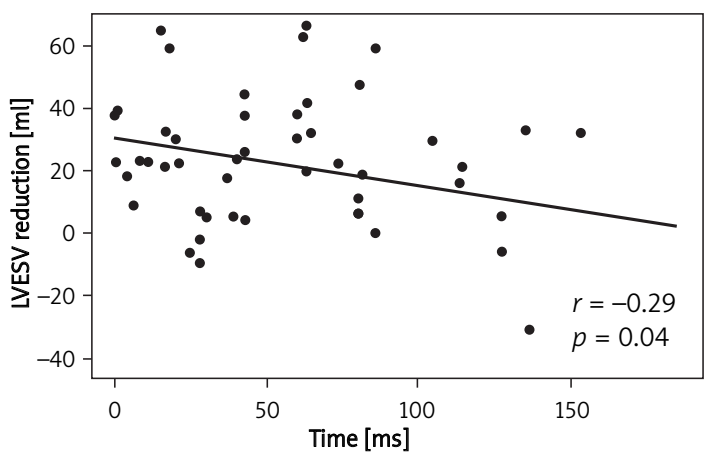

B

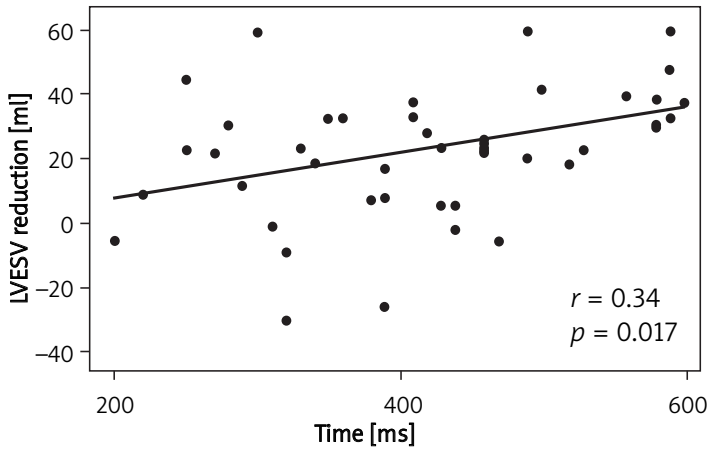

Figure 1. Correlation between the magnitude of LVESV reduction following CRT introduction and initial parameters of mechanical dyssynchrony: A - T TDI basal LV-RV segments, $B-T_{\text {strain }}$ of 12 LV segments

$T_{T D I}$ basal $L V-R V$ segments - maximum difference of time from the beginning of QRS complex to maximum systolic velocity between basal left ventricle and right ventricle segments, $T_{\text {strain }}-12$ - maximum difference of time from the beginning of QRS complex to maximum systolic strain among 12 left ventricle segments 
represented by the assessment of longitudinal left ventricle shortening velocities using colour-coded TDI from the apical window [15]. We found no significant correlation of the analysed colour-coded TDI parameters of intraventricular dyssynchrony with LVESV reduction. Similar results come from other studies on correlations of TDI baseline indices with LVESV volume changes after CRT. Miyazaki et al. failed to demonstrate the usefulness of septallateral, anteroseptal-posterior delay and $\mathrm{T}_{\mathrm{TDI}}{ }^{-} \mathrm{SD}$ in a median 6-month-follow-up [24]. Phillips et al. found no predictive value of septal-lateral delay [25].

The interventricular dyssynchrony assessed with maximal difference between time from the beginning of QRS complexes to the peak velocity of basal LV and RV segments correlated negatively, indicating that the smaller the initial difference the more pronounced the reverse remodelling after CRT, which is quite an interesting finding. The echocardiographic assessment of interventricular dyssynchrony in previous studies on CRT was mainly based on the interventricular mechanical delay index (IVMD). The IVMD, reproducible and easily calculated in daily practice had little efficacy in predicting response to CRT as compared to intraventricular dyssynchrony assessment [26-29]. It should also be noted that evaluation of interventricular dyssynchrony with TDI and evaluation with IVMD represent different phenomena of heart performance: TDI, the movement of heart muscle parts; IVMD, the blood flow. The timing of the ejection of blood out of ventricles is conditioned by many factors including valves' and right ventricle function, different haemodynamic states, pulmonary diseases, etc., and poorly reflects the synchrony of myocardial contraction. The role of interventricular dyssynchrony and the optimal approach to the issues of TDI parameters are yet to be clarified.

\section{Advantage of myocardial strain calculation. Strain rate imaging inapplicable}

Strain and strain rate imaging, thought to be unaffected by movement of structures in the thorax, adjoining parts of heart muscle or the location in the ventricle, have the theoretic advantage of differentiating active myocardial contraction from passive translational movement [30]. Unfortunately, they are difficult to calculate in spherical LV geometry common in CHF, their application requires proper training and experience of the operator as well as good quality images, and they have disputable reproducibility [15].

Advantages of strain imaging in the prediction of CRT outcome were emphasized by some researchers [31-33]. Breithardt et al. concluded that the degree of dyssynchrony was not accurately represented by the timing of myocardial velocity, especially in ischaemic heart disease, and proposed that the timing of deformation should be the preferred modality [31]. Sogaard et al. found that the extent of delayed longitudinal contraction at the base predicted LVEF increase following CRT [32, 33]. In our study the only strain parameter that differentiated responders from non-responders and correlated with the magnitude of LVESV reduction was $T_{\text {strain }}-12$. In the aforementioned study of Miyazaki, baseline $T_{\text {strain }}$-SD showed a significant correlation with the percent reduction in LVESV at the follow-up $(r=0.57, p<0.001)$ [24]. This supports the idea of eliciting the sites of the earliest and the latest activation with the strain imaging technique.

Parameters of strain rate in our study were not related to the change of LVESV. This is coherent with the ASE report based on the series of the studies of Yu et al., who failed to demonstrate the usefulness of strain rate imaging to predict the LV reverse remodelling response to $\mathrm{CRT}[34,35]$.

The multivariate analysis showed that the predictive significance of dyssynchrony appraisal is lost when other variables are taken into account. This emphasizes the role of complex evaluation of potential CRT recipients including a broad array of conditions associated with poor prognosis in CHF [1].

Our study group, after inclusion of patients with atrial fibrillation and the group of pacemakers upgraded to CRT, was heterogeneous but highly reflected the clinical profile of patients undergoing biventricular stimulator implantation in daily practice. Recording myocardial walls separately reduced Doppler angle dependent bias in calculating echocardiographic parameters, but caused that analysed curves, though always at least 3 were considered, came from different heart cycles.

The study had a non-randomized design, the sample size was small and the observational period was relatively short. Clinical and heart performance changes elicited by CRT may not always appear simultaneously and patients who present with clinical effects may not exhibit echocardiographic improvement and vice versa; thus longer follow-up would definitely provide accessory information about the significance of left ventricle reverse remodelling noted 3 months after CRT introduction [36].

In conclusion, only a few TDI-derived parameters such as maximum time difference from the beginning of QRS complex to maximum strain among 12 basal and midlevel left ventricular segments or maximum time difference from the beginning of QRS complex to maximum myocardium velocity in colour-coded TDI between left and right ventricular basal segments can be useful to predict the magnitude of left ventricle reverse remodelling after CRT introduction. The predictive value of the parameters is limited when other clinical and biochemical factors are considered. 


\section{Acknowledgments}

The study was supported by the KBN grant 2 P05B 02330.

\section{References}

1. Dickstein K, Cohen-Solal A, Filippatos G, et al. ESC guidelines for the diagnosis and treatment of acute and chronic heart failure 2008: the Task Force for the diagnosis and treatment of acute and chronic heart failure 2008 of the European Society of Cardiology. Developed in collaboration with the Heart Failure Association of the ESC (HFA) and endorsed by the European Society of Intensive Care Medicine (ESICM). Eur J Heart Fail 2008; 10: 933-89.

2. Dickstein K, Vardas PE, Auricchio A, et al. 2010 focused update of ESC Guidelines on device therapy in heart failure: an update of the 2008 ESC Guidelines for the diagnosis and treatment of acute and chronic heart failure and the 2007 ESC Guidelines for cardiac and resynchronization therapy. Developed with the special contribution of the Heart Failure Association and the European Heart Rhythm Association. Eur J Heart Fail 2010; 12: 1143-53.

3. Adamson PB, Kleckner KJ, VanHout WL, Srinivasan S, Abraham WT. Cardiac resynchronization therapy improves heart rate variability in patients with symptomatic heart failure. Circulation 2003; 108: 266-9.

4. Lappegard KT, Bjornstad H. Anti-inflammatory effect of cardiac resynchronization therapy. Pacing Clin Electrophysiol 2006; 29: 753-8.

5. Sarzi Braga S, La Rovere M, Pedretti R. Baroreflex sensitivity normalization after cardiac resynchronization therapy (Case report). Int J Cardiol 2006; 109: 118-20.

6. Simantirakis EN, Schiza SE, Siafakas NS, Vardas PE. Sleepdisordered breathing in heart failure and the effect of cardiac resynchronization therapy. Europace 2008; 10: 1029-33.

7. Vardas PE, Auricchio A, Blanc JJ, et al. Guidelines for cardiac pacing and cardiac resynchronization therapy: the task force for cardiac pacing and cardiac resynchronization therapy of the European Society of Cardiology. Developed in collaboration with the European Heart Rhythm Association. Eur Heart J 2007; 28: 2256-95.

8. Gorcsan J 3rd, Oyenuga O, Habib PJ, et al. Relationship of echocardiographic dyssynchrony to long-term survival after cardiac resynchronization therapy. Circulation 2010; 122: 1910-8.

9. Yu CM, Bleeker GB, Fung JW, et al. Left ventricular reverse remodeling but not clinical improvement predicts longterm survival after cardiac resynchronization therapy. Circulation 2005; 112: 1580-6.

10. Di Biase L, Auricchio A, Sorgente A, et al. The magnitude of reverse remodelling irrespective of aetiology predicts outcome of heart failure patients treated with cardiac resynchronization therapy. Eur Heart J 2008; 29: 2497-505.

11. Elliott P. Cardiomyopathy. Diagnosis and management of dilated cardiomyopathy. Heart 2000; 84: 106-12.

12. Elliott $P$, Andersson $B$, Arbustini $E$, et al. Classification of the cardiomyopathies: a position statement from the European Society Of Cardiology Working Group on Myocardial and Pericardial Diseases. Eur Heart J 2008; 29: 270-6.

13. Bailey R, Shepard R, Hsu K, Zmijewski M, Roberts J, Sambelashvili A. Optimization of CRT timing by fusion in
ECG lead V1 is associated with a higher increase in leftventricular contractility. Circulation 2007; 116: II_554-5.

14. Evangelista A, Flachskampf F, Lancellotti P, et al. European Association of Echocardiography recommendations for standardization of performance, digital storage and reporting of echocardiographic studies. Eur J Echocardiogr 2008; 9: 438-48.

15. Gorcsan J 3rd, Abraham T, Agler DA, et al. Echocardiography for cardiac resynchronization therapy: recommendations for performance and reporting: a report from the American Society of Echocardiography Dyssynchrony Writing Group endorsed by the Heart Rhythm Society. J Am Soc Echocardiogr 2008; 21: 191-213.

16. Wiliński J, Czarnecka D, Wojciechowska W, et al. Clinical and classic echocardiographic features of patients with and without left ventricle reverse remodeling following cardiac resynchronization therapy introduction. Cardiol J 2011; 18: 157-64.

17. Wiliński J, Czarnecka D, Wojciechowska W, et al. Different response rates to cardiac resynchronization therapy (CRT) according to the applied definition. Prz Lek 2009; 66: 130-3.

18. Notabartolo D, Merlino JD, Smith AL, et al. Usefulness of the peak velocity difference by tissue Doppler imaging technique as an effective predictor of response to cardiac resynchronization therapy. Am J Cardiol 2004; 94: 817-20.

19. Shen X, Aronow WS, Anand K, et al. Evaluation of left ventricular dyssynchrony using combined pulsed wave and tissue Doppler imaging. Arch Med Sci 2010; 6: 519-25.

20. Rajdev A, Aronow WS, Lai HM, et al. Comparison of left ventricular ejection fraction by single photon computed tomographic myocardial perfusion imaging versus coronary computed tomography angiography. Arch Med Sci 2009; 5: 28-31.

21. Upadhyay GA, Choudhry NK, Auricchio A, Ruskin J, Singh JP. Cardiac resynchronization in patients with atrial fibrillation: a meta-analysis of prospective cohort studies. J Am Coll Cardiol 2008; 52: 1239-46.

22. Tada $\mathrm{H}$, Toide $\mathrm{H}$, Okaniwa $\mathrm{H}$, et al. Maximum ventricular dyssynchrony predicts clinical improvement and reverse remodeling during cardiac resynchronization therapy. Pacing Clin Electrophysiol 2007; 30 Suppl 1: S13-8.

23. Delnoy PP, Ottervanger JP, Luttikhuis $\mathrm{HO}$, et al. Comparison of usefulness of cardiac resynchronization therapy in patients with atrial fibrillation and heart failure versus patients with sinus rhythm and heart failure. Am J Cardiol 2007; 99: 1252-7.

24. Miyazaki C, Lin G, Powell BD, et al. Strain dyssynchrony index correlates with improvement in left ventricular volume after cardiac resynchronization therapy better than tissue velocity dyssynchrony indexes. Circ Cardiovasc Imaging 2008; 1: 14-22.

25. Phillips KP, Popovic ZB, Lim P, et al. Opposing wall mechanics are significantly influenced by longitudinal cardiac rotation in the assessment of ventricular dyssynchrony. JACC Cardiovasc Imaging 2009; 2: 379-86.

26. Achilli A, Peraldo C, Sassara M, et al. Prediction of response to cardiac resynchronization therapy: the selection of candidates for CRT (SCART) study. Pacing Clin Electrophysiol 2006; 29 Suppl 2: S11-9.

27. Ghio S, Constantin C, Klersy C, et al. Interventricular and intraventricular dyssynchrony are common in heart failure patients, regardless of QRS duration. Eur Heart J 2004; 25: $571-8$.

28. Richardson M, Freemantle N, Calvert MJ, Cleland JG, Tavazzi L Predictors and treatment response with cardiac 
resynchronization therapy in patients with heart failure characterized by dyssynchrony: a pre-defined analysis from the CARE-HF trial. Eur Heart J 2007; 28: 1827-34.

29. Chung ES, Leon AR, Tavazzi L, et al. Results of the Predictors of Response to CRT (PROSPECT) trial. Circulation 2008; 117: 2608-16.

30. Marwick TH. Measurement of strain and strain rate by echocardiography: ready for prime time? J Am Coll Cardiol 2006; 47: 1313-27.

31. Breithardt OA, Stellbrink C, Herbots L, et al. Cardiac resynchronization therapy can reverse abnormal myocardial strain distribution in patients with heart failure and left bundle branch block. J Am Coll Cardiol 2003; 42: 486-94.

32. Sogaard P, Egeblad H, Kim WY, et al. Tissue Doppler imaging predicts improved systolic performance and reversed left ventricular remodeling during long-term cardiac resynchronization therapy. J Am Coll Cardiol 2002; 40: 723-30.

33. Sogaard P, Hassager C. Tissue Doppler imaging as a guide to resynchronization therapy in patients with congestive heart failure. Curr Opin Cardiol 2004; 19: 447-51.

34. Yu CM, Fung JW, Zhang Q, et al. Tissue Doppler imaging is superior to strain rate imaging and postsystolic shortening on the prediction of reverse remodeling in both ischemic and nonischemic heart failure after cardiac resynchronization therapy. Circulation 2004; 110: 66-73.

35. Yu CM, Gorcsan J 3rd, Bleeker GB, et al. Usefulness of tissue Doppler velocity and strain dyssynchrony for predicting left ventricular reverse remodeling response after cardiac resynchronization therapy. Am J Cardiol 2007; 100: 1263-70.

36. Bax JJ, Abraham T, Barold SS, et al. Cardiac resynchronization therapy: Part 1 - issues before device implantation. J Am Coll Cardiol 2005; 46: 2153-67. 